\title{
Indirect Holography Breast Cancer Detection System (IH-BCDS) Using VORD
}

\author{
Sardar Asad Ali Biabani \\ Researcher, Science and Technology Unit \\ Umm Al-Qura University, Makkah Al-Mukarramah, Saudi Arabia
}

Emad Shafie

Vice Dean for Academic Affairs, Makkah Community College

Umm Al-Qura University, Makkah Al-Mukarramah, Saudi Arabia

\section{Prof. Mohamed Osama Khozium}

Department of Engineering \& Applied Science - Computers

Umm Al-Qura University, Makkah Al-Mukarramah, Saudi Arabia

doi: 10.19044/esj.2017.v13n9p10 $\quad$ URL:http://dx.doi.org/10.19044/esj.2017.v13n9p10

\begin{abstract}
Microwave Imaging is a prominent technique in current imaging modalities for breast cancer detection and its treatment, primarily due to the significant dielectric property contrast between normal and malignant breast tissues. This study describes how VORD Viewpoint-Oriented Requirement Definition (VORD) method is used to examine patients for early detection of cancer by microwave imaging by realizing the existence of multiple perspectives by terminating differences in the demands of stakeholders.

The proposed system in this paper is based on microwave imaging by Indirect Holography for Breast Cancer Detection System (IH-BCDS) using Viewpoint-Oriented Requirement Definition (VORD) method. The suggested approach for developing such system is to investigate the possibility of detecting tumors at an early stage by utilizing VORD as analysis tool which is an activity oriented framework for IH-BCDS requirement elicitation. If the system gets implemented successfully, it can play a vital role in detecting the tumor at an early stage by helping a medical specialist in decision making to diagnose the patient.
\end{abstract}

Keyword: Analysis, Microwave Imaging, Mammography, Patient, Requirement elicitation

\section{Introduction}

Microwave imaging technique is firmly based on recovering the electrical properties, namely permittivity, and conductivity, of materials. 
Microwave imaging for biomedical applications is especially holding the attention because the properties of a dielectric range of tissues can give significant information about their health (Golnabi, Meaney, Geimer \& Paulsen, 2011). In the paper, the imaging technique taken up is the approach known as Microwave Indirect Holographic technique serving as an essential component is a synthesized reference beam. Eldson Et.al proposed a novel microwave imaging approach for early detection of breast cancer, which engages in the use of Microwave Indirect Holographic technique applying a patented synthetic reference wave. This approach offers benefits regarding simplicity, expense, comfort and safety when compared to current mammography technologies (Elsdon, Yurduseven \& Smith, 2013). This approach was initially developed for determining the complex aperture fields of antennas without using vector measurement equipment (Smith, Leach, Elsdon \& Foti, 2007) and subsequently extended for imaging of metallic objects (Yurduseven, Smith, Livingstone, Schjbal \& You 2013). For metal object detection, it was noticed that the image could be rebuilt by taking amplitude measurements with a power meter. A large number of women are affected by breast cancer, and detecting it at an early stage and applying medical procedures at opportune is the key to long-term survival and life quality for patients (Cancer facts and figures, 2003). X-ray is the modern gold standard for imaging of breast cancer (Brown, Houn, Sickles \& Kessler, 1995). By contrast, it has flaws like missing approximately $10-30 \%$ of breast cancers. Another flaw of X-ray mammography is the inconvenience women with dense breast deal during imaging (Nass, Henderson \& Lashof, 2001). Also, X-ray ionizing properties restrict the number of observations of screening. Such limitations motivate the researchers to come up with new methods for detection of breast tumor. Diagnostic techniques like ultrasound and MRI of the breast, which does not uncover the patient to ionizing radiation, are used for the detection of breast cancer. Ultrasound of the breast is weighted as an appropriate modality for the initial evaluation of a palpable breast mass in pregnant women under the age of 30 ("ACR Standards", 2000-01), ("National Comprehensive Cancer Network", 1998).

Kotonya and Somerville proposed the Viewpoints Oriented Requirements Definition (VORD) as a technique to tackle engineering requirements from a level of viewpoint (Kotonya and Sommerville, 1996). Designing, testing, and programming is not an essential part of the development of software, but requirement analysis. Identifying all the needs of the client whose operations connects a degree of user interaction is a problem for the interactive system. The expert has to make sure that the needs of the clients get approved at the same time in an accurate way. VORD is useful in detecting the stakeholder needs and also recognizing the services that a user anticipates from the system (Pozgaj, 2000). To determine 
viewpoints and their requirements, VORD provides a structured technique for analyzing, collecting and documenting. Viewpoints map classes of endusers of a system or to other systems interfaced to it. Direct viewpoints are the viewpoints that make up the core model. Indirect viewpoints are the viewpoints concerned with the systems influence on the organization which allows organizational demands and concerns (Kotonya, 1997). The creators of the VORD identified a restriction that it does not explicitly support the interaction analysis across and within all of the viewpoints.

This paper presents a proposed system based on microwave imaging by Indirect Holography for Breast Cancer Detection System (IH-BCDS) using Viewpoint-Oriented Requirement Definition (VORD) method in the analysis stage and has six sections. The first section is the introduction. The second section is about related work. The third section talks about the background of Indirect Holography. The fourth section discusses an overview of VORD. In the fifth section, we will analyze and review the proposed system. The sixth section concludes the paper and suggests future work.

\section{Related Works}

As per American Cancer Society (ACS), one of the serious health problem in the U.S are breast cancer and about 40,000 women were at a risk of losing their life due to it by 2009. In the U.S breast cancer is most commonly diagnosed beside skin cancer and is rated as the second highest mortality rate of cancer in women after lung cancer. $3.2 \%$ per year decrease in breast cancer mortality in women younger than 50 years of age based on the stats taken from 1990 to 2006 (Breast Cancer Facts and Figures, 200910). Early detection of breast cancer and improvement in treatment response time and monitoring is the result of this decline. The standard clinical procedure is X-ray mammography to detect abnormalities in the breast. The increase in the level of fibro-glandular tissue in women with high-density breast may be less visible or unclear the presence of a tumor, which in result the responsiveness of mammography can be well below normal (Buist, Porter, Lehman, Taplin \& White, 2004). From the health care managers perspective, the false positive rate of X-ray mammography can lead to surgical intervention which is expensive and unnecessary. MRI (Magnetic Resonance Imaging), US (Ultrasound), CT (Computerized Tomography) and $\mathrm{X}$-ray are available imaging techniques for breast cancer screening in the clinics which may be sensitive than mammography in selected population suffer from low specificity and fail to provide functional information the change in the molecular level of the tissue.

Golnabi et al. illustrate in their paper, the most recent clinical microwave imaging with $2 \mathrm{D}$, and $3 \mathrm{D}$ reconstructed images of the phantom from different experiments and patients record. Eldson et al. present in their 
paper an alternative approach for breast cancer detection using microwave imaging which is upon indirect microwave holographic approach, central to which is the use of a synthetic reference beam. The theoretical basis for microwave breast imaging is the high contrast between the permittivity and conductivity of healthy and malignant breast tissue. This contrast is made available in a variety of studies (Joines, Jirtl, Rafal \& Schaefer, 1980), (Joines, Shrivastav \& Jirtle, 1989), (Chaudhary, Mishra, Swarup \& Thomas, 1984), (Campbell and Land, 1992), (Joines, Zhang, Li \& Jirtle, 1994), (Lazebnik et al., 2007).

(Ahmed and Khozium, 2008) illustrate in their paper the using of VORD methodology in analyzing Medical care system.

(Araujo and Coutinho, 2003) proposed a paper on the VISION model. Their approach took ideas from the VORD and PREVIEW (Sommerville, Sawyer \& Viller, 1998) models, then formed viewpoint associations, UML modeling, and aspectual use cases. They have recognized some basic techniques of identifying and documenting viewpoint relationships that we would like to incorporate into our enhancement.

Zeljka Pozgaj used the VORD process model during requirement analysis in which he perceptible give details of the three steps of the VORD using a Stakeholder example. The UML diagrams illustrate the viewpoint and service interactions (Pozgaj, 2000). They are other techniques of incorporating multiple viewpoints into requirements engineering other than the VORD model. Lee's Proxy Viewpoints Model-based Requirements Discovery (PVRD) (Lee, 2002) methodology is for working on "legacy" SRS documents.

As the matter is sensitive and related to breast cancer screening in women, it requires privacy and security during analyzing and designing the proposed system by using hiding techniques (Khozium, 2016), (Abuarafah and Khozium, 2008), (Khozium, 2016).

\section{Background of Indirect Holography}

There is an originating body of literature on medical applications of microwave imaging (Golnabi et al.). Techniques like dynamic microwave imaging exploit contrast in dielectric properties or backscatter methods. The objective of microwave tomography is to recover the properties of the dielectric profile of an object that is from the microwave energy measurements transmitted through the object (Smith et al.), (Breast Cancer Facts and Figures, 2009-10). The initially obtained results of the breast imaging are promising (Joines et al.), (Chaudhary et al.).

Yurduseven et al. describe in their paper how we can implement indirect holography for imaging passive objects. It gives us details of complex scattered field values which can be obtained in an easy and less 
expensive way from measurements that is taken by sampled scalar intensity over a single scanning aperture.

(Sheen, McMakin \& Hall, 2001) in their paper describe how images of scattering objects can be obtained using back-propagation techniques from knowledge of the complex field at the measurement plane.

\section{Overview of VORD}

VORD is a method based on viewpoints. The model used is a service-oriented one, where the viewpoints are considered to be clients if one was to think of it as a client-server system (Kotonya and Sommerville, 1996).

A viewpoint in VORD receives service from the system and in turn, passes control information to the network. The service oriented approach makes VORD suited for specifying interactive systems (Kotonya and Sommerville, 1998). VORD has two types of viewpoints

Direct

Indirect

Direct viewpoints receive services from the system and send control information and data to the system.

Indirect viewpoints do not directly interact with the system, but it has an interest in some or all of the services delivered by the system.

For any medium or large size systems, different types of end-user are there. Various stakeholders have a different interest in the requirements of the system. The problem can be seen in multiple ways by different viewpoints. However, their perspectives are not totally free from external control and constraint but usually, coincide to have common requirements. Viewpoint key strength is- oriented analysis which recognizes the existence of many perspectives and gives a framework for determining the existence of conflicts in the requirements proposed by different stakeholders. Viewpoints in VORD method is deemed to be as service receivers. In this scenario, viewpoints are external to the system and receive services from the system (Kotonya and Sommerville, 1996). Viewpoints for these services may provide data. The analysis involves investigating the services received by different points of view, which collects them and resolve their conflicts. Interactive systems deliver services to end-users. As a consequence, the most valid viewpoint - oriented idea for interactive systems analysis uses external views. The viewpoints interact with the system by receiving services from it and provide data to the system.

\section{VORD Method Advantages}

There is a natural way to structure the needs of elicitation process as Viewpoints are external to the system. If a point of view is valid, it is 
relatively easy to decide. Viewpoints must interact with the system somehow. Viewpoints and services are useful methods to structure nonfunctional requirements. Each service may make a logical connection to nonfunctional requirements. The same service is allowed by multiple viewpoints to have separate non-functional requirements in different points of view. The VORD method has been designed as a service-oriented framework for requirements elicitation and analysis.

\section{VORD Method Principal Stages}

VORD method principle stages as shown in figure 1 is viewpoint identification, structuring, documentation, and mapping. Determining viewpoints that receive system services and identifying particular services provided to each point of view is Viewpoint identification. Viewpoint structuring involves grouping related viewpoints into a hierarchy. Joint activities are provided at levels in the hierarchy and are inherited by lowerlevel viewpoints. Viewpoint documentation calls for refining the description of the viewpoints identified and services. Viewpoint-system mapping, which involves identifying, objects in an object-oriented design using services information, which is encapsulated in viewpoints. (Pozgaj, 2000), (Araujo and Coutinho, 2003)

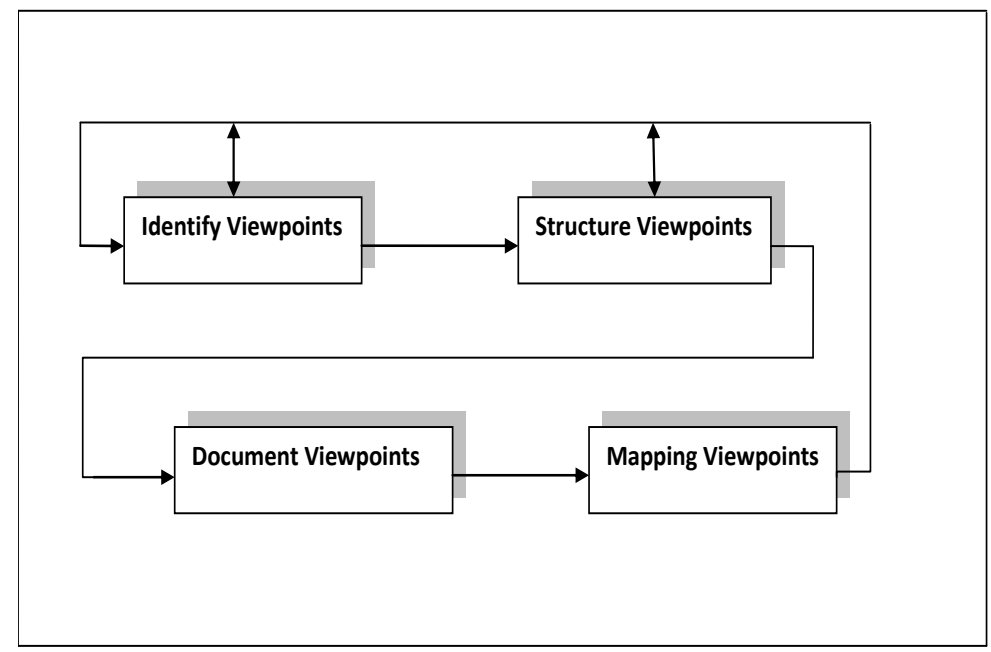

Fig 1: The Principle Stages of VORD Method

The VORD method guides the medical practitioner in identifying viewpoints. It provides a number of theoretical viewpoints which act as a starting point for identification. The VORD method has been chosen as an activity oriented framework for IH-BCDS elicitation and analysis. 


\section{Proposed System}

The proposed system is grouped and structured in the hierarchical form which represents the activities for each viewpoint. Figure 2 shows the main structure of the proposed system of IH-BCDS.

\section{Viewpoints in IH-BCDS}

\section{The Oncologist}

$\mathrm{He}$ is a specialist in oncology who is responsible for the study and treatment of tumors and to provide online help to the system admin and other stakeholders.

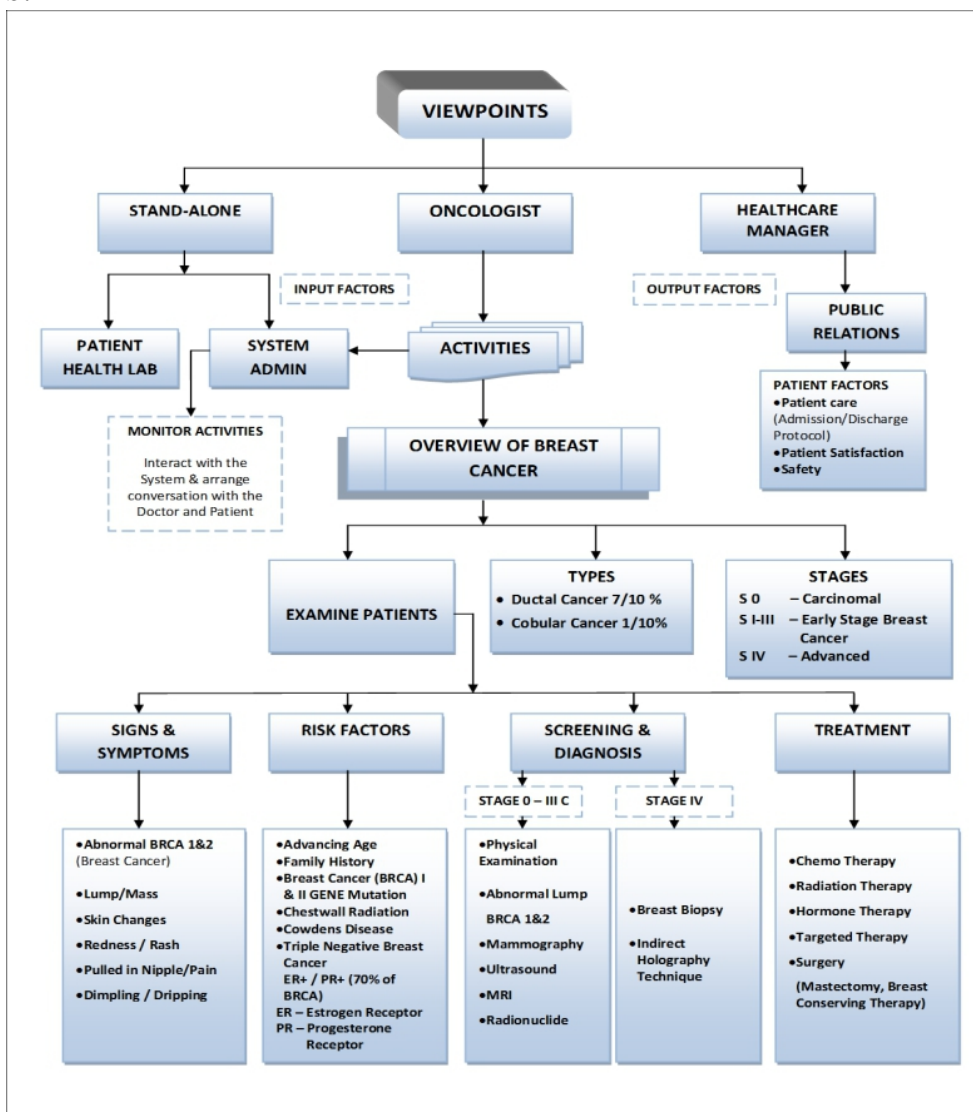

Figure 2: Main Structure of Indirect Holography Breast Cancer Detection System (IH-BCDS)

\section{Stand-alone}

It is an electronic system which is capable of operating instructions received from the oncologist independently and will revolve around patient health lab and system admin that retrieve necessary data from the system to perform stand-alone operations. 


\section{System Admin}

$\mathrm{He}$ is the person responsible for monitoring the activities of the treatment by interacting with the system and arranging a conversation with the doctor and the patient.

\section{Healthcare Manager}

$\mathrm{He}$ is in charge of overseeing the operations of the treatment given, with the help of system admin and focuses on the output patient factors with an aim to maintain a strong public relationship.

\section{Architecture}

The proposed system architecture consists of four main factors. Figure 3 illustrates the main architecture of the proposed system.

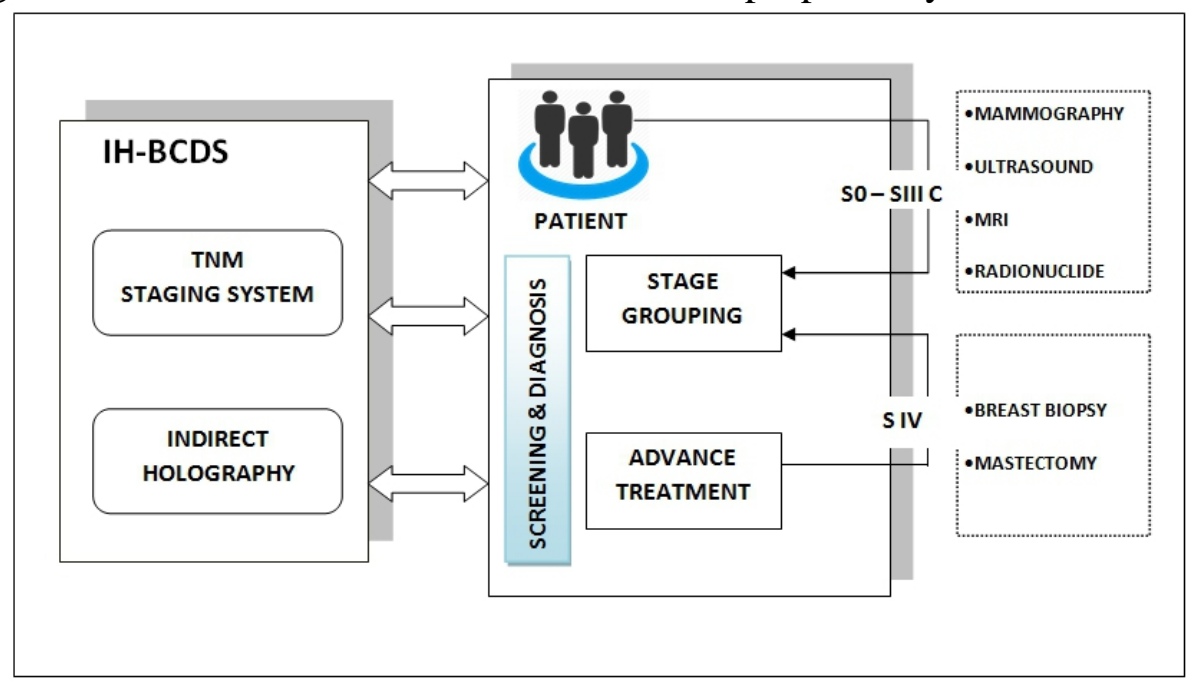

Figure 3: Architecture of Indirect Holography Breast Cancer Detection System (IH-BCDS)

IH-BCDS includes mainly two decision-making modules: TNM (Classification of Malignant Tumors) staging system and Indirect Holography.

\section{TNM Staging System}

Classification of Malignant Tumors (TNM) is a staging system in which the stage of the cancer is identified that originates from the tumor with alphanumeric codes.

$\mathrm{T}$ describes the size of the primary tumor whether it has invaded nearby tissue.

$\mathrm{N}$ describes lymph nodes that are involved in the nearby region. 

body).

M describes metastasis (spreading of cancer to another part of the

IH-BCDS acquires knowledge from three sources.

Test the patient by VORD to define the initial diagnosis.

Study the case by stage grouping to perform the screening and diagnosis.

If the tumor lies between Stages 0-III c diagnostic tests can be Mammography/ MRI/Ultrasound depending on the evidence of malignancy.

\section{IH-BCDS Inference}

The main rule in $\mathrm{IH}-\mathrm{BCDS}$ is to determine the result of each patient based on the evidence of its malignancy stage. If the tumor lies between stage 0-IIIc it goes for the initial treatment i.e. Mammography/Ultrasound, and if it is in stage IV, it instructs the system to go for the advance treatment.

\section{Indirect Holography}

Indirect Holography is performed if the tumor is in stage IV, the viewpoint decision is to go for advance treatment i.e. Breast Biopsy or Mastectomy.

\section{Conclusion and Future work}

This paper discusses IH-BCDS which diagnose early breast cancer with the sequence of VORD. The proposed system will help the Oncologist in decision making with the aid of viewpoints to examine the condition of the patient by group staging and to diagnose the patient depending on its stages rather than opting for the advanced treatment initially.

The proposed system will be developed according to the need of the stakeholders by focusing the patient factors. The use of this system will offer substantial benefits regarding simplicity and expense when compared to other techniques. As of future work, we would like to include MATLAB software in our system to simulate the tumor and to study the measurements between the original and reconstructed images of breast phantom. We can suggest using multi-agent systems approach by adding databases, functionalities, and scenarios (Biabani and Khozium, 2013), (Khozium, 2012-13).

By transferring viewpoints into agents and extract the following:

Goal Refinement.

Goal Rearrangement and grouping.

Functionalities interactions with databases to extract and define agents.

Scenario development.

Percepts, actions, and protocols.

System overview diagram including actions, percepts, and protocols. 


\section{References:}

1. Golnabi, A. H., Meaney, P. M., Geimer, S., \& Paulsen, K. D.(2011) Microwave imaging for breast cancer detection and therapy monitoring. IEEE Topical Conference on Biomedical Wireless Technologies, Networks, and Sensing Systems. doi: 10.1109/BIOWIRELESS.2011.5724347

2. Elsdon, M., Yurduseven, O., \& Smith, D. (2013) Early Stage Breast Cancer Detection using Indirect Microwave Holography. Progress In Electromagnetics Research,143, 405-419. doi: 10.2528/PIER13091703

3. Smith, D., Leach, M., Elsdon, M., \& Foti, S. J. (2007) Indirect holographic techniques for determining antenna radiation characteristics and imaging aperture fields. IEEE Antennas and Propagation Magazine, 49, (1), 54-67. doi: 10.1109/MAP.2007.370982

4. Yurduseven, O., Smith, D., Livingstone, B., Schejbal, V., \& You, Z. (2013) Investigations of resolution limits for indirect microwave holographic imaging. International Journal of $R F$ and Microwave Computer-Aided Engineering, 23, (4), 410-416. doi: 10.1002/mmce. 20727

5. Cancer facts and figures. (2003). American Cancer Society. Retrieved from http://www.cancer.org/research/cancer-factsstatistics/all-cancer-facts-figures.html

6. Brown, M. L., Houn, F., Sickles, E. A., \& Kessler, L. G. (1995) Screening mammography in community practice: positive predictive value of abnormal findings and yield of follow-up diagnostic procedures. American Journal of Roentgenology, 165, 1373-1377. doi: 10.2214/ajr.165.6.7484568

7. Nass, S.J., Henderson, I.C., \& Lashof, J.C. (2001) Committee on Technologies for the Early Detection of Breast Cancer, Mammography, and Beyond: Developing Technologies for the Early Detection of Breast Cancer Eds. National Cancer Policy Board, Institute of Medicine, and Commission on Life Studies, National Research Council.

8. ACR Standard for the Performance of Breast Ultrasound Examinations ( 2000-2001) ACR Standards Reston, Va: American College of Radiology 389-392.

9. National Comprehensive Cancer Network (1998) Practice Guidelines: screening for and evaluation of suspicious breast lesions. NCCN Proceedings. Oncology, 12, (11A) 89- 138. 
10. Kotonya, G., \& Sommerville, I. (1996) Requirements Engineering with Viewpoints. Software Engineering Journal, 11, (1), 5-18. doi: 10.1049/sej.1996.0002

11. Pozgaj, Z. (2000) Requirement Analysis Using VORD, 22nd International Conference. Information Technology Interfaces, Pula, Croatia. University of Zagreb, Croatia.

12. Kotonya, G. (1997) Practical Experience with viewpoint-Orientated Requirements Specification Requirements Engineering Journal, 4, (1), 115-133. doi:10.1007/s007660050023

13. Breast Cancer Facts \& Figures (2009-2010) American Cancer Society. from http://www.cancer.org/Research/CancerFactsFigures/Breast CancerFactsFigures/f861009-final-9-08-09-pdf.

14. Buist, D. S. M., Porter, P. L., Lehman, C., Taplin. S. H., \& White, E. (2004) Factors contributing to mammography failure in women aged 40-49 years. Journal of the National Cancer Institute, 96, (19), 1432-1440. doi: https://doi.org/10.1093/jnci/djh269

15. Joines, W.T., Jirtle, R.L., Rafal, M.D., \& Schaefer, D.J. (1980) Microwave power absorption differences between normal and malignant tissue. International Journal of Radiation Oncology Biology Physics,6,(6), 681-687. doi: http://dx.doi.org/10.1016/03603016(80)90223-0

16. Joines, W.T., Shrivastav, S., \& Jirtle, R.L. (1989) A comparison using tissue electrical properties and temperature rise to determine the relative absorption of microwave power in malignant tissue. The International Journal of Medical Physics Research and Practice, 16, (6), 840-844. doi: $10.1118 / 1.596308$

17. Chaudhary, S.S., Mishra, R.K., Swarup, A. \& Thomas, J.M. (1984) Dielectric properties of normal \& malignant human breast tissues at radiowave \& microwave frequencies. Indian J Biochem Biophys, 21, (1), 76-79.

18. Campbell, A. M., \& Land, D.V. (1992) Dielectric properties of female human breast tissue measured in vitro at $3.2 \mathrm{GHz}$. Physics in Medicine and Biology, 37, (1), 193-210.

19. Joines, W.T., Zhang, Y., Li, C., \& Jirtle, R.L. (1994) The measured electrical properties of normal and malignant human tissues from 50 to $900 \mathrm{MHz}$. Medical Physics, 21, (4), 547-550. doi: 10.1118/1.597312

20. Lazebnik, M., Watkins, C.B., Hagness, S.C., Booske, J.H., Popovic, D., McCartney, L., Okoniewski, M., Lindstrom, M.J., Breslin, T.M., Harter, J., Sewall, S., Temple, W., Mew, D., Magliocco, A., \& Ogilvie, T. (2007) The dielectric properties of 
normal and malignant breast tissue at microwave frequencies: analysis, conclusions, and implications from the Wisconsin/Calgary study. In Proc. IEEE Antennas and Propagation International Symposium, 2172-2175. doi: 10.1109/APS.2007.4395958

21. Ahmad, F., \& Khozium, M. O. (2008) Medical Care System using VORD Methodology, Ubiquitous Computing and Communication Journal, 3,(1), 68-74.

22. Araújo, J., \& Coutinho, P. (2003) Identifying Aspectual Use Cases Using a Viewpoint-Oriented Requirements Method, Early Aspects 2003: AspectOriented Requirements Engineering and Architecture Design. Workshop of the 2nd International Conference on AspectOriented Software Development, Boston, USA.

23. Sommerville, I., Sawyer, P., \& Viller, S. (1998) Viewpoints for Requirements Elicitation: A Practical Approach. IEEE International Conference on Requirements Engineering, 74-81.

24. Lee, S. W. (2002) Proxy Viewpoints Model-based Requirements Engineering. ACM Symposium on Applied Computing 1004-1008. doi: $10.1145 / 508791.508986$

25. Sheen, D. M., McMakin, D. L., \& Hall, T.E. (2001) Threedimensional millimeter-wave imaging for concealed weapon detection. IEEE Transactions on Microwave Theory and Techniques 49, (9), 1581- 1592. doi: 10.1109/22.942570

26. Kotonya, G., \& Sommerville I. (1998) Requirements Engineering: Process and Techniques. Wiley, United Kingdom. (Chapter 5, 6)

27. Khozium, M. O. (2016) Security Using Hiding Technique: EOB Case Study. International Journal in IT and Engineering, 4, (3) 18-33.

28. Abuarafah, A. G., \& Khozium, M. O., (2008) Proposed Structure for High-Level Security Enhancement. Ubiquitous Computing and Communication Journal, 3,(3), 137-142.

29. Khozium M. O. (2006) Indicators Classification for Software Assessment. Egyptian Computer Science journal, 28, (2).

30. Biabani S. A., \& Khozium M.O. (2013) Multi-Agent RFID Process In Project Chain Management. International Journal of Management, IT and Engineering, 3, (4), 539-549.

31. Khozium, M. O. (2013) Multi-Agent System Overview: Architectural Designing using Practical Approach. International Journal Of Computers \& Technology, 5, (2) 85-93.

32. Khozium M. O. (2012) A Hybrid Intelligent Information System for the Administration of Massive Mass of Hajjis. Life Science Journal, 9, (2), 377-383. 